
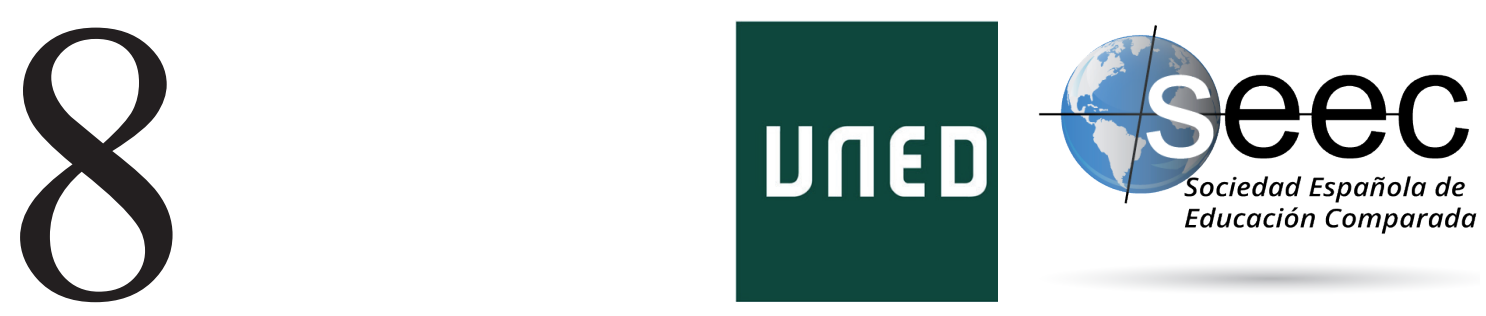

\title{
Segregación social y equidad educativa en Chile y Argentina
}

Social segregation and educational equity in Chile and Argentina

\section{Daniel Pedro Míguez*; Jason Beech**; Malena Nigro***}

DOI: $10.5944 /$ reec.40.2022.28951

\section{Recibido: 23 de noviembre de 2020 Aceptado: 5 de julio de 2021}

\footnotetext{
*Daniel Pedro Míguez: investigador principal y profesor titular. Instituto de Geografía, Historia y Ciencias Sociales. Consejo Nacional de Investigaciones Científicas y Técnicas. Universidad Nacional del Centro de la Provincia de Buenos Aires. Datos de contacto: E-mail: dpmiguez@gmail.com

**JASON BEeCH: senior lecturer, Faculty of Education, Monash University. Datos de contacto: E-mail: jason.beech@monash.edu

***Malena Nigro: estudiante de posgrado. Universidad de San Andrés. Datos de contacto: E-mail: malenanigro@gmail.com
} 


\title{
Resumen
}

El propósito de este artículo consiste en comparar los niveles de segregación social en los sistemas educativos de Chile y Argentina y su relación con el acceso equitativo al saber escolarizado en ambos países. Complementariamente, nos proponemos explorar si las políticas de financiamiento de la educación orientadas por la lógica de libre mercado aplicadas en Chile, generaron niveles excepcionales de segregación social en comparación con Argentina, país en el que las políticas de financiación educativa preservaron la centralidad de la educación pública y gratuita. El análisis se realiza mediante el uso de brechas percentílicas en base a los datos PISA 2012. Se estiman los niveles de segregación según el nivel educativo de los padres, el porcentaje de estudiantes en las escuelas con mayores niveles de segregación y la asociación entre los niveles de segregación y el desempeño de los estudiantes en las Pruebas. El estudio muestra patrones de segregación complejos. En Chile los grados de segregación son mayores, pero la proporción de población en escuelas con alta segregación es baja y hay mayor equidad en la distribución del conocimiento. En Argentina hay menores grados de segregación, pero la proporción de la población en escuelas con alta segregación es mayor y hay mayor heterogeneidad en el acceso al conocimiento. Los patrones de segregación encontrados muestran matices que no confirman plenamente la hipótesis de partida. En este sentido, sugieren que la segregación social se daría con o sin mecanismos de mercado. Estos últimos no necesariamente introducen mayores niveles de inequidad y las formas y niveles de desigualdad varían según los aspectos o dimensiones del sistema educativo que se consideren.

Palabras clave: segregación; desempeño; equidad; mercado; Chile; Argentina.

\begin{abstract}
The purpose of this article is to compare the levels of social segregation in the educational systems of Chile and Argentina and their relationship with equitable access to schooled knowledge in both countries. Based on this comparison, we will explore if the policies based on a 'free market' implemented in Chile generated exceptional levels of social segregation and inequalities in access to school knowledge when compared to Argentina, where education policies have retained the principle of public and free education with no selectivity. The analysis is carried out through the use of percentile gaps based on PISA 2012 data. The levels of segregation are estimated according to the educational level of parents, the percentage of students in schools with higher levels of segregation and the association between the levels of segregation and the performance of the students in the Tests. Result: the study shows complex segregation patterns. In Chile the degrees of segregation are higher, but the proportion of the population in highly segregated schools is low and there is greater equity in the distribution of knowledge. On the other hand, in Argentina there are lower degrees of segregation, but the proportion of the population in highly segregated schools is higher and there is greater heterogeneity in access to knowledge. The patterns of segregation found show nuances that do not fully confirm the initial hypothesis, but rather suggest that social segregation would occur with or without market mechanisms, that the latter do not necessarily introduce higher levels of inequity and that the forms and levels of inequality vary according to the aspects or dimensions of the educational system that are considered.
\end{abstract}

Keywords: segregation; achievement; equity; Chile; Argentina. 


\section{Introducción}

El propósito de este artículo consiste en comparar los niveles de segregación social en los sistemas educativos de Chile y Argentina y su relación con el acceso al saber escolarizado en ambos países. Aunque existen antecedentes de estudios comparativos sobre los niveles de segregación social en los sistemas educativos de Chile y Argentina, estos aún dejan interrogantes. Como veremos, se han realizado estudios internacionales que incluyen a ambos países y algunas comparaciones específicas entre ellos, aunque de carácter más bien exploratorio. En la mayor parte de los casos, estos solo consideran la segregación según el estrato social de pertenencia de los estudiantes. Si bien en casi todos los estudios se propone un vínculo entre ésta y el acceso diferencial al saber escolarizado, en las investigaciones comparativas ese vínculo, rara vez, se examina empíricamente. Además, si bien la mayoría de los trabajos estiman los grados o niveles de segregación social, no establecen las proporciones de la población estudiantil que es afectada por ella (es decir, el porcentaje de estudiantes que asiste a escuelas donde hay sectores sociales fuertemente sobre-representados en comparación con su distribución en el conjunto de la población). Así, en este artículo pretendemos realizar una contribución a este campo de estudios examinando las complejas articulaciones que se producen cuando se estiman las diversas proporciones de estudiantes que son afectados por la segregación social en cada uno de los países y cómo ésta se asocia al acceso diferencial al saber escolar de los y las estudiantes.

Si bien este será el objetivo central de este trabajo, complementariamente nos proponemmos examinar una premisa que ha orientado una buena cantidad de los estudios comparativos. Esta es que las políticas de financiamiento de la educación aplicadas en Chile generarían niveles excepcionales de segregación social, ya que han permitido la selección de estudiantes según su capacidad económica y académica (mediante los sistemas de vouchers, 'copago' público/privado y admisión que explicamos luego). Según esta postura, se debería constatar una diferencia significativa con la Argentina. Donde, si bien existieron matices en las políticas de financiación educativa, éstas preservaron la centralidad de la educación pública y gratuita; y desde 1984 deshabilitaron la selección de estudiantes según criterios académicos, al menos en las escuelas estatales. Así, la existencia de mayores niveles de segregación y desigualdad en el acceso al saber en Chile, sin confirmarla definitivamente, serían compatibles con esta postura. Mientras que la inexistencia de desigualdades significativas o si éstas fueran mayores en Argentina, sin invalidarla plenamente, tenderían a relativizarla.

En este trabajo, los cálculos respecto a los niveles de segregación social y de acceso al saber escolarizado están basados en datos de las pruebas PISA 2012. Elegimos este año porque permite examinar los niveles de segregación social y acceso diferencial al saber mientras estaban vigentes las políticas de financiamiento educativo aplicadas en Chile. Ya que desde el año 2015 se instrumentaron cambios en las formas de financiación y selección de estudiantes que tenían por objetivo moderar sus efectos (más detalles luego). De esa manera, las estimaciones que realizamos registran los niveles de segregación y acceso diferencial al conocimiento mientras las políticas estaban vigentes y antes de que empezara un proceso de transformación de las mismas.

Comenzaremos nuestro análisis presentando los avances existentes en este campo de investigación. Luego, describiremos las variables y el método utilizados. Posteriormente expondremos los grados y patrones de segregación social y el acceso diferencial al saber 
escolar que surgen del análisis de estas variables. En las conclusiones sintetizaremos los principales resultados alcanzados tomando en cuenta la importancia de incluir la relación entre segregación social y desempeño académico en este tipo de estudios.

\section{Segregación social y educación}

La segregación social puede definirse como la distribución desigual de estudiantes de distinto estrato social al interior del sistema educativo (Dupriez, 2010; Maroy, 2004). Es decir, se produce segregación cuando la concentración de alumno/as de un mismo sector social en ciertas escuelas difiere significativamente de la del promedio del sistema. Se ha señalado, además, que la segregación social suele estar asociada a una distribución inequitativa del conocimiento escolarizado debido a la diferencia en la disponibilidad de recursos (de infraestructura y humanos) entre escuelas que concentran población de distintos estratos sociales (Albornoz, 2016; Bellei, 2013; Valenzuela, 2008). De esta forma, la investigación existente sugiere que sistemas educativos con altos grados de segregación social afectaran el acceso igualitario al conocimiento escolarizado, reproduciendo las diferencias sociales de origen.

Los estudios que se han detenido en los factores que promueven o generan la segregación social entre escuelas señalan que estos pueden ser tanto internos como externos al sistema educativo (Bellei, 2013). Los primeros incluyen los exámenes de admisión, el costo de la educación privada o también factores culturales como las formas de sociabilidad y o los hábitos conductuales 'distintivos' de un sector social (estética personal, las formas de habla o modalidades de interacción social, etc.). Los segundos refieren a aspectos tales como las preferencias parentales o la segregación residencial, los niveles de ingreso familiar o la inserción laboral de los progenitores.

Aunque no podemos detenernos en esto aquí, al considerar los posibles factores exógenos es oportuno señalar la diversidad de procesos socioeconómicos que atravesaron Chile y Argentina en las últimas décadas. Cómo lo muestran Miguez y Dewey (2017) desde la década de 1980 Chile ha tenido un crecimiento sostenido de su PBI y experimentado una reducción sistemática de la pobreza. Y aunque se ha mantenido como un país más desigual que la Argentina, ha experimentado una reducción sistemática de su coeficiente de Gini. Además, su tasa de graduación de la educación secundaria se ha expandido. Los datos de SITEAL ${ }^{1}$ indican que la proporción de la población adulta que concluyó el ciclo aumentó del 45,7 \% en el año 2000 al 61,4 \% en 2015. A diferencia de Chile, en el caso de Argentina, se produjo un estancamiento económico prolongado, con crecimiento de la pobreza y la desigualdad. A esto se suma una tasa de graduación de la educación secundaria más baja que la chilena. Nuevamente, los datos de SITEAL indican que ésta evolucionó del 42,4 \% en el 2000 al 56,5 \% en el 2015. Dentro de esta tendencia general existen, por supuesto, diferencias por sector etario y estrato social. Entre la población de 20 a 24 años, en Argentina la tasa pasó del 56,8 \% en el 65,3 \% en ese período. En Chile el incremento fue mayor, evolucionó del 65,2 al 85,7 por ciento. Asimismo, en el $30 \%$ de la población de menores ingresos en Argentina la tasa de graduación pasó del 19,6 al 39,9 por ciento en el período 2000-2015; mientras que en Chile se incrementó del 30,6 al 52,6 por ciento.

Ahora bien, si entre los factores exógenos se destaca una evolución diferencial de las condiciones económicas y sociales, desde el punto de vista de los factores endógenos,

1 https://siteal.iiep.unesco.org/indicadores 
se destacan los contrastes en las políticas de financiamiento educativo. Desde la década de 1980, Chile desarrolló un sistema de 'libre mercado' basado en el financiamiento de la demanda educativa a través de vouchers. En este sistema las familias pueden elegir las escuelas, mientras el Estado aporta un pago mensual por cada estudiante indistintamente del sector educativo, público o privado, al que pertenezca la escuela. Asociado a esto, desde 1993 se permitió a las escuelas cobrar un 'copago' adicional al aporte realizado por el Estado, lo que supuso condicionar las posibilidades de elección al status socioeconómico de las familias. Asimismo, a partir de 1995 comenzaron a difundirse los resultados por escuela de las pruebas estandarizadas nacionales (SIMCE), con el objetivo de incentivar aún más la selección de las mismas según su calidad académica. De esta manera, hacia finales de la década de 1990, el sistema educativo chileno se presentaba como ampliamente selectivo, ya sea por condiciones socioeconómicas, como por criterios culturales y académicos. ${ }^{2}$

En contraste con el sistema educativo chileno, en el argentino, más allá de una oscilante expansión del sector privado que describimos luego, la mayor parte de la educación siguió siendo pública y gratuita, tanto en el nivel primario como secundario Los datos de SITEAL indican que en el año 2015 en torno al 74,8 \% de los estudiantes del nivel secundario asistían a la educación pública y en torno al 26 \% a la educación privada, valores que no se modifican significativamente desde la década de 1990 (Narodowski y Moschetti, 2015). Esto contrasta con el caso chileno, donde en el año 2000 el 54,5 \% de la población asistía al sector público, proporción que se redujo al 40,1 \% en 2015.

A su vez, a diferencia del caso chileno, en Argentina la política oficial no fomentó las prácticas de admisión selectivas, particularmente desde la restauración democrática de 1983. De hecho, desde 1984 se eliminaron los exámenes de ingreso en las escuelas de nivel nacional para evitar la selectividad según capacidades, aunque en rigor y dado el carácter federal del sistema educativo, esto no eliminó totalmente las prácticas selectivas. Como lo indican Rigal et al. (2019), la tendencia a restringir la selectividad mediante exámenes y otros mecanismos de admisión por capacidad académica se fue profundizando progresivamente. Sin embargo, en algunas jurisdicciones y establecimientos educativos persistieron mecanismos selectivos. En una minoría de casos, se mantuvieron los exámenes de ingreso para escuelas con sobre demanda. También se mantuvieron, entre otras formas de selección, la asignación de prioridades a hermanos o hermanas de estudiantes ya ingresados a la escuela, o la selección por orden de inscripción, que favorece a los sectores con mayor acceso a la información (Veleda, 2014). En definitiva, si bien en Argentina las normativas tendieron a desalentar los mecanismos selectivos, algunos de ellos persistieron debido a la permanencia de prácticas consuetudinarias y la heterogeneidad de normativas jurisdiccionales.

Las particulares características del sistema educativo chileno lo han hecho objeto de crecientes investigaciones sobre el efecto de sus políticas de libre mercado educativo sobre los niveles de segregación social (Ascorra et al., 2016; Contreras, et al., 2010; Murillo, 2016; Treviño et al., 2016; Valenzuela, 2008). Como adelantamos, la premisa sobre la que se han basado la mayor parte de los estudios es que los sistemas de voucher, copago y admisión mediante exámenes de ingreso o entrevistas de selección han generado

2 A partir de los años 2015 y 2017 se sancionaron normativas modificando el sistema de vouchers y copagos para reducir el nivel de selectividad del sistema (ver Falabella, 2015 y Matelluna, 2019 para una descripción más detallada del proceso de reformas y de las implicancia de las nuevas normas). 
niveles excepcionales de segregación social en el sistema educativo chileno. Y que estos se han vinculado a un desigual acceso al saber escolarizado. Si bien presentan matices, en general los estudios sobre el caso chileno han tendido a confirmar estas premisas. Por ejemplo, Valenzuela (2008) ha mostrado que si se considera al $10 \%$ de los estudiantes de nivel socioeducativo más alto, Chile ocupa el segundo lugar mundial en los niveles de segregación en su sistema educativo (junto a Brasil y después de Tailandia). Y, además, llega al primer lugar si se considera al primer tercil en la escala de nivel económico social, aunque los resultados son algo más moderados entre los deciles intermedios (Valenzuela et al., 2013).

Asimismo, un estudio de Contreras et al. (2010) muestra que, en promedio, un 32 \% de los estudiantes chilenos atravesaron algún proceso de selección para ingresar a la escuela y que este porcentaje se incrementaba en las escuelas privadas subsidiadas. Allí, el $48 \%$ de los estudiantes eran seleccionados de acuerdo a su desempeño académico, $26 \%$ por criterios socioeconómicos y $20 \%$ por criterios religiosos. A su vez, Bellei (2013) confirmó estos resultados, mostrando que la segregación social en el sistema educativo respondía más a la selección por habilidades y a la política de copago que a la situación residencial. Es decir que algunas de las investigaciones que examinaron los efectos de las políticas de libre mercado educativo en Chile confirmaron que el sistema de copago y de selección de estudiantes se asociaba a importantes grados de segregación social.

Complementariamente, otras investigaciones revelaron mecanismos adicionales que fomentaban la segregación. Por ejemplo, Verger et al. (2016) pusieron en evidencia que las familias seleccionan aquellos establecimientos que reúnen estudiantes del mismo sector social, evitando los que congregan alumnos de estratos diferentes (particularmente los más bajos). Este criterio predominaba, incluso, por sobre el del rendimiento académico expresado en pruebas estandarizadas que alentaban las políticas educativas. Así, y atendiendo a estas características de la demanda, las escuelas implementaban mecanismos selectivos que más que apuntar a incrementar el desempeño académico, se orientaban a preservar la composición social del estudiantado.

Finalmente, Flores et al. (2013) encontraron que además de responder a las restricciones impuestas por el sistema de copago y la selección académica, la segregación se asociaba también a diferencias culturales. Aun controlando la incidencia de los mecanismos de selección por copago y la influencia de la cercanía residencial, el estudio revelaba que las familias de niveles socioculturales más bajos optaban por escuelas de menor rendimiento académico. Así, a la vez que el estudio confirmaba que las restricciones económicas y la selectividad académica restringían la libertad de elección de las familias, también mostraban que ésta respondía a las expectativas surgidas de su constitución cultural.

En suma, la investigación en el caso de Chile ha constatado la existencia de altos niveles de segregación social asociada a las políticas de libre mercado educativo que habilitan los sistemas de copago y dispositivos de admisión mediante, entrevistas, exámenes de ingreso o antecedentes de desempeño académico. Aunque también ha encontrado otros mecanismos más sutiles, relacionados a los criterios culturales de selección y a las redes sociales de pertenencia.

En el caso de Argentina la investigación no ha tenido el mismo desarrollo que en Chile. Algunos estudios recientes se han enfocado en el problema de la segregación en el sistema educativo y han producido diversas mediciones del fenómeno. Sin embargo, salvo excepciones (Albornoz, 2016; Kruger, 2014, 2019), la investigación no ha aprovechado los datos provistos por las pruebas estandarizadas, ya sea nacionales o internacionales. 
En lugar de ello han utilizado los datos de la Encuesta Permanente de Hogares (EPH), que no incluye información sobre las escuelas a las que concurren los estudiantes o sobre su desempeño educativo. De manera tal que, en general, los estudios han analizado la segregación en función del nivel socioeconómico de la población, pero no han examinado su vínculo con el desempeño académico, ni estimado las posibles morfologías de la segregación más allá de la distinción entre sector público y privado.

Un estudio pionero en este sentido ha sido el de Narodowski y Nores (2002) que compara Argentina y Chile. El estudio constata altos niveles de segregación en Chile en base a los resultados de las pruebas estandarizadas de nivel nacional. Sin embargo, en base a los datos de la EPH de Argentina observan que, desde los años '6o, se produjo un incremento del enrolamiento de los sectores de mayores ingresos en la educación privada produciendo una creciente segregación. Si bien la diversidad de fuentes no les permite a los autores llegar a conclusiones definitivas, los resultados generan dudas sobre las reales diferencias entre ambos países y sugieren que la segregación social se daría con o sin los mecanismos de selección por ingresos o por desempeño académico.

Los estudios posteriores sobre la segregación en Argentina no retomaron la perspectiva comparativa, pero sí analizaron la evolución de la matrícula en el sector público y privado según el nivel de ingreso de las familias. Los resultados de estos trabajos han sido matizados. Por ejemplo, examinando el comportamiento de la matricula desde 1980, Gasparini et al., (2011) encuentran que la segregación, entendida como el mayor enrolamiento relativo en el sector privado, crece en períodos expansivos y se contrae en los ciclos recesivos de la economía. En un estudio posterior Narodowski y Moschetti (2015) arribaron a conclusiones similares, observando un crecimiento del enrolamiento en el sector privado entre mediados de la década de 1990 y el año 2010, pero con fluctuaciones según los ciclos recesivos o expansivos de la economía. En el mismo sentido, tanto González Rozada, et al. (2015), como Martín (2017) encuentran que las familias en los quintiles de ingreso más altos tienden a inscribir a sus hijos en el sector privado. Aunque en general no constatan que esto resulte en niveles muy marcados de segregación. Así, la investigación en este campo sugiere una cierta tendencia a que los sectores de mayores ingresos prefieran la educación privada en los casos en que les resulte accesible. Pero más que derivar en incrementos o decrecimientos sostenidos de la segregación, esto resulta en ciclos de expansión o contracción de la misma según la situación económica del País.

Notablemente, los estudios de Krüger (2014, 2019), aunque se basan en fuentes alternativas (las pruebas PISA), arriban a resultados compatibles con los anteriores. En su estudio de 2014 la autora muestra que los sectores ocupacionales de menor calificación tienden a estar sobre-representados en el sector público y los de mayor calificación en el sector privado. Sin embargo, nota también que esta situación varía según las circunstancias económicas. Así, al comparar los años 2000 y 2009 observa una creciente heterogeneidad (menos segregación) en el sector privado. Esto ocurre asociado a una evolución favorable de los indicadores de nivel económico social que permite a los sectores menos calificados acceder a la educación paga (Kruger, 2014). Esta tendencia se verifica también en su estudio de 2019 al comparar la evolución de la segregación social entre los distintos países de América Latina entre 2000 y 2015. Allí la autora observa una caída del $2 \%$ en los niveles de segregación en el caso de Argentina, contrario al caso chileno donde crece entre tres y ocho puntos porcentuales cada tres años durante el lapso considerado (Kruger, 2019).

En suma, la mayor parte de la investigación para el caso argentino sugiere que pese a 
que operaron ciertos mecanismos de segregación social, particularmente entre el sector privado y público, ésta no habría llegado a niveles relativamente altos, ni experimentado un crecimiento sostenido y homogéneo en el conjunto del sistema. De esta manera, la comparación entre la mayor parte de la investigación llevada adelante en Chile y Argentina tiende a confirmar la premisa de que los sistemas de libre mercado educativo se asocian a niveles de segregación mayores que aquellos que, más allá de los matices, siguen otorgando un rol central al sector público.

Sin embargo, estas investigaciones no establecen la proporción de la población que asiste a las escuelas donde se produce la segregación en cada uno de los países, ni tampoco comparan las diferencias en el acceso al conocimiento escolarizado que éstas generan. Además, en el caso argentino, la mayoría de las investigaciones solo estiman la segregación según el nivel socioeconómico de la población que asiste al sector público o privado, pero no examinan las formas de la segregación más allá de esta dicotomía. En lo que sigue describiremos las variables y métodos que emplearemos para intentar dar cuenta de estos aspectos aún no estudiados de la segregación, y luego discutiremos los resultados que surgen de ellos.

\section{Métodos y Variables}

En este estudio estimaremos los niveles de segregación según el método de brechas percentílicas (Gasparini et al., 2011; Jaume, 2013). ${ }^{3}$ Este método permite una buena descripción de los grados de segregación social en el interior de cada uno de los sistemas educativos, aunque en general no se ha aplicado para realizar un análisis 'causal' de la misma (Gasparini et al., 2011; Jaume, 2013; Martin, 2017). Debido a ello, nos limitaremos a estimar las formas y grados de segregación social que encontremos en Chile y Argentina y su relación con el acceso al saber escolarizado. También examinaremos la proporción de estudiantes afectados por la segregación social y las desigualdades en el acceso al saber. Si bien, como adelantamos, no podremos establecer un nexo causal entre estas estimaciones y las políticas de financiamiento educativo en uno y otro país, sí podremos ver si las diferencias entre Chile y Argentina son compatibles con la postura que sostiene que los sistemas cuyas politícas de financiamiento educativo se basan en una lógica de libre mercado se asocian a niveles excepcionales de segregación social y desigualdad en el acceso al saber escolarizado. Es decir, no podremos construir modelos estadísticos que muestran vínculos causales en un sentido estricto. Pero sí ver hasta qué punto los patrones de segregación y desigualdad en uno y otro país presenta las diferencias que supone esta premisa.

La variable que utilizamos para estimar las diferencias sociales fue el del máximo nivel educativo alcanzado por los progenitores. Si bien las bases PISA 2012 incluyen múltiples variables proxi del nivel social de los estudiantes (como el tipo de inserción laboral de los progenitores, disponibilidad de bienes durables o culturales en el hogar, etc.), el máximo nivel educativo alcanzado por los progenitores se mostró como la variable con mayor capacidad discriminante. Es decir, resultó la variable que permitía discernir mejor los niveles y formas de segregación social entre distintos grupos de estudiantes en cada país.

3 Preferimos esta aproximación a otros indicadores más usados, como el Indice de Disimilitud (Duncan y Duncan, 1955) o el Indice de Aislamiento (Lieberson, 1981), ya que estos presentan limitaciones en situaciones en las que existen patrones de segregación complejos como los de Chile y Argentina (Kruger, 2019). 
Dado que esta variable se presenta en las bases desagregada a nivel individual, construimos una nueva variable estimando la cantidad de progenitores en cada nivel educativo por escuela. Para evitar sesgos en los niveles de representatividad, eliminamos aquellas escuelas donde hubieran contestado menos de 10 estudiantes; lo que nos dejó un total de 195 escuelas para Chile y 216 para Argentina. Sobre esta base calculamos el porcentaje de progenitores con secundaria terminada en cada escuela. Esto nos permitió estimar la segregación social entre escuelas según la concentración de progenitores con o sin secundaria terminada.

Para simplificar la presentación de los datos, dividimos esta escala en cinco intervalos equivalentes - quintiles - según el porcentaje de progenitores con secundaria terminada (de o a $20 \%$, de 20,01 al 40 \%; del 40,01 al 60 \%; del 60,01 al 80 \% y del 80,01 al 100 \%). Esto nos permitió estimar, además, la proporción de estudiantes en cada intervalo de la escala. Para facilitar la comparación entre países, también transformamos esta medida en una nueva escala con media o y desviación estándar 1 ('puntajes z'). Así pudimos reconocer los grados de segregación según la 'distancia' (proporción de desviaciones) que separaba a cada escuela y a cada rango de la distribución media de cada sistema educativo -ya sea el de Chile o de Argentina.

Para estimar el acceso al conocimiento escolarizado utilizamos el puntaje alcanzado por los estudiantes en las pruebas PISA. Ciertamente, esta es una medida limitada del acceso al saber, ya que las Pruebas miden algunas formas específicas de conocimiento, que además pueden contener sesgos culturales (Meyer y Benavot, 2013; Rivas, 2015). Sin embargo, pese a estas limitaciones, los resultados en las Pruebas siguen siendo una variable proxi del acceso a un saber que favorece la integración social -el acceso al mercado laboral, el ejercicio efectivo de los derechos ciudadanos, etc. (Duro et al., 2010). De manera que, si bien no deben desconocerse sus limitaciones, es claro que la variable basada en el puntaje obtenido por los estudiantes es una buena aproximación de su acceso a un saber a su vez escolarizado y socialmente relevante. ${ }^{4}$

Los puntajes obtenidos por los estudiantes en las Pruebas se presentan desagregados por áreas de conocimiento (matemática, ciencia y lectura). En este caso, estimamos los puntajes obtenidos por los estudiantes como el promedio de los valores alcanzados en ellas. Además, como los datos se presentan desagregados por estudiante, también debimos estimar los promedios por escuela y observar la desviación estándar en cada una para asegurarnos niveles aceptables de representatividad. Elegimos utilizar el promedio de los resultados obtenidos en cada una de las áreas temáticas, ya que los puntajes se encontraban fuertemente asociados entre sí (Alfa de Cronbach: 0,95). Esto nos permitía construir una medida global del desempeño que resultaba más adecuada a nuestros propósitos, ya que no era el objeto de este estudio examinar las diferencias entre áreas temáticas.

Esto nos permitió construir una escala que oscilaba entre el menor puntaje promedio obtenido por una escuela y el mayor de ellos. La escala se extiende de 152 a 542 puntos en Argentina y de 322 a 592 puntos en Chile. La diferencia entre escalas no solo indica un mejor desempeño en Chile, sino que la mayor extensión de la escala en Argentina

4 Existe una fuerte crítica respecto a, entre otras cosas, la 'validez’ de las pruebas PISA (la adecuación de sus variables para medir capacidades distintas en contextos culturales diversos) y a la imposibilidad de las pruebas de estimar la verdadera capacidad de los participantes de 'aplicar' el conocmiento que se manifiesta en ellas (Carabaña, 2015; Pereyra et al., 2013). Aunque no es posible dar cuenta del complejo debate aquí, aún quienes aceptan estas críticas sugieren que más que descartar totalmente los datos, conviene considerarlos aunque controlando sus sesgos (Martinez García, 2016). 
sugiere también una mayor inequidad en el acceso al conocimiento en este segundo país. Como en el caso anterior, agrupamos estas escalas en cinco intervalos equivalentes, para ver la proporción de la población que se concentraba en las escuelas de mayor y menor puntaje. Además, también construimos una nueva escala con puntajes z para facilitar la comparación entre países.

Finalmente, estimamos la relación entre la segregación social y la distribución del saber escolarizado observando los promedios de puntaje obtenido por las escuelas pertenecientes a cada uno de los rangos según la proporción de padres con secundaria terminada. De esta manera pudimos establecer el nivel de asociación entre la segregación social y la distribución del saber escolarizado. En este caso también construimos escalas con puntajes z para facilitar la comparación entre países.

\section{Resultados}

Los primeros datos que presentamos en esta sección (Tabla 1) indican los niveles de segregación entre escuelas según la proporción de padres con secundario terminado en cada establecimiento. Posteriormente mostraremos las diferencias en el acceso al saber escolarizado según los puntajes obtenidos en las pruebas PISA (Tabla 2). Y finalmente daremos cuenta de los grados y formas de asociación entre ambas variables (Tabla 3).

En lo que respecta a la segregación social, un primer dato significativo es que Chile y Argentina presentan diferencias en la proporción de padres con secundaria concluida entre escuelas. La primera columna de la Tabla 1 refleja estas diferencias según las desviaciones estándar que separan a cada grupo del promedio del sistema educativo de cada país. Esta estimación muestra que, además de existir diferencias en los grados de segregación, los patrones de segregación también son distintos.

Tabla 1.

Segregación según Nivel Educativo del Padre

\section{Columna 1}

Columna 2

\begin{tabular}{|c|c|c|c|c|}
\hline \multirow[b]{2}{*}{ Escala } & \multicolumn{2}{|c|}{ Desviaciones a la media } & \multicolumn{2}{|c|}{ Porcentaje de estudiantes } \\
\hline & Argentina & Chile & Argentina & Chile \\
\hline & $-1,59$ & $-2,18$ & & \\
\hline $0-20$ & $(14,1 / 5,8)$ & $(14,7 / 3,4)$ & 8,48 & 4,95 \\
\hline & $-0,79$ & $-1,44$ & & \\
\hline $20,1-40$ & $(32 / 5,3)$ & $(34,5 / 4,9)$ & 28,71 & 9,31 \\
\hline & o,o3 & $-0,78$ & & \\
\hline 40,1 a 60 & $(50 / 5,4)$ & $(52 / 8,7)$ & 27,81 & 16,35 \\
\hline & 0,81 & $-0,09$ & & \\
\hline 60,1 a 80 & $(68 / 5,5)$ & $(70,3 / 5,7)$ & 18,00 & 20,81 \\
\hline & 1,68 & 0,86 & & \\
\hline 80,1 a 100 & $(87,9 / 74,5)$ & $(95,5 / 5,4)$ & 17,01 & 48,58 \\
\hline
\end{tabular}

Fuente: base PISA 2012. Promedio por quintil y desviación típica entre paréntesis; p= o,ooo. 
En el caso de Chile, vemos que los quintiles más bajos son, a su vez, los que más se apartan del promedio. En este caso, el primer quintil se encuentra a más de dos desviaciones por debajo de la media, mientras que el segundo está a casi una vez y media de la misma. A su vez, los rangos más altos presentan una menor separación. El quintil cinco no llega a una desviación estándar de diferencia, mientras que el cuatro se encuentra a menos de una décima por debajo de la misma. Es decir que en Chile la segregación social supone grupos de población muy por debajo del promedio de escolarización que se concentran en algunas escuelas, y una mayoría de estudiantes cercanos a la media o moderadamente por encima de ella que se concentra en otras.

Si bien en Argentina también existen importantes niveles de segregación, esta se distribuye de manera más simétrica. Mientras que en Chile los rangos más bajos de la escala se encuentran muy distantes de la media, en Argentina las escuelas que concentran población de sectores más y menos escolarizados se distribuyen 'equilibradamente' en torno a ella. Así, los quintiles uno y cinco se encuentran a aproximadamente 1,5 desviaciones estándar por debajo y por encima de la media respectivamente. Y los quintiles 2 y 4 se encuentran a algo más de media desviación estándar de ésta, mientras que el 3 se ubica casi exactamente en la media. De esta forma, en lugar de encontrar un grupo de escuelas altamente segregadas en uno de los extremos de la escala, encontramos una distribución equilibrada en torno a los valores intermedios de la misma.

Las razones de esta distribución pueden comprenderse cuando se analizan las proporciones de estudiantes en los distintos quintiles (la Columna 2 del Gráfico) donde surgen, nuevamente, importantes diferencias entre países. Chile muestra una concentración de la población en los quintiles más altos. En torno al 70 \% de los estudiantes concurre a escuelas donde más del $60 \%$ de los padres concluyeron la escuela secundaria (quintiles 4 y 5). De hecho, casi el 50 \% de la población concurre a escuelas donde más del $80 \%$ de los padres concluyó ese nivel educativo y solo el $15 \%$ de los estudiantes asiste a establecimientos donde menos del $40 \%$ de los padres lo hizo. Esto es congruente con los resultados anteriores, ya que la concentración de estudiantes en los rangos más altos de la escala resulta en que estos se encuentren más cerca del promedio poblacional.

En el caso de Argentina, la población no se concentra en los rangos más altos, sino en los medios y medio bajos. El $56 \%$ de los estudiantes concurren a escuelas donde menos del 60 \% y más del $20 \%$ de los padres concluyó la educación secundaria (quintiles 2 y 3). Sólo el $17 \%$ concurre a escuelas donde más del $80 \%$ de los padres concluyeron este nivel educativo y un $18 \%$ adicional lo hace a establecimientos donde más del 60 pero menos del 80 por ciento lo hizo. A su vez, solo el 8,4 \% de los estudiantes concurre a escuelas donde menos del 20 \% concluyó el nivel medio. Esto es compatible con la distribución más simétrica de la población en torno al promedio, ya que la mayor parte se encuentra en los rangos intermedios en lugar de concentrarse en uno de los polos como en Chile.

En suma, la comparación entre Chile y Argentina revela dos patrones de segregación social distintos. En Chile se observa un sector ampliamente diferenciado del conjunto, con menor nivel de escolarización pero que concentra a porcentajes de la población relativamente bajos. Mientras tanto, la mayor parte de la población asiste a escuelas en las cuales los progenitores alcanzan niveles de escolarización que se encuentran en torno al promedio o por encima de él. En la Argentina, los niveles o grados de diferenciación por sector social son menores, pero la mayor parte de la población estudiantil se encuentra en los rangos que concentran a población con niveles de escolarización medio o medio bajos. Puesto de manera más sintética: en Chile existen mayores niveles o grados de 
segregación que afectan negativamente (se encuentran por debajo de la media) a sectores relativamente minoritarios de estudiantes; en Argentina los niveles de segregación son menores, pero afectan negativamente a una mayor proporción de estudiantes.

Ahora bien, además de estimar la segregación social en este estudio calculamos el acceso diferencial al saber escolarizado según los puntajes obtenidos en las pruebas PISA. La tabla 2 pone en evidencia los resultados de esta última estimación, agrupando las escuelas según el puntaje promedio obtenido en las pruebas. En este caso se hace necesario agrupar las escuelas de manera ligeramente diferente en Argentina y Chile, ya que los puntajes promedio difieren entre un país y otro. Como señalamos, los puntajes en Chile tienden a ser más altos que en Argentina y las diferencias entre rangos de puntajes tienden a ser menores. Lo que sugiere un menor desempeño y más desigual en la Argentina que en Chile.

Tabla 2.

Nivel de Desempeño en las Pruebas Pisa

\begin{tabular}{|c|c|c|c|c|c|}
\hline \multirow[t]{2}{*}{ Escala Chile } & $\begin{array}{c}\text { Desviaciones } \\
\text { a la } \\
\text { media }\end{array}$ & \multirow[t]{2}{*}{$\begin{array}{c}\text { Porcentaje } \\
\text { de } \\
\text { Estudiantes }\end{array}$} & Escala Argentina & $\begin{array}{l}\text { Desviaciones a } \\
\text { la media }\end{array}$ & \multirow[t]{2}{*}{$\begin{array}{l}\text { Porcentaje } \\
\text { de } \\
\text { Estudiantes }\end{array}$} \\
\hline & $-1,22$ & & & $-3,36$ & \\
\hline 322 a 376 & $(358 / 14,8)$ & 4,39 & 152 a 230 & $(183 / 43)$ & 0,70 \\
\hline & $-0,69$ & & & $-1,87$ & \\
\hline 376,01 a 430 & $(400 / 16,2)$ & 32,98 & 230,01 a 308 & $(278 / 20,6)$ & 3,11 \\
\hline & 0,006 & & & $-0,72$ & \\
\hline 430,01 a 484 & $(456 / 14,5)$ & 27,92 & 308,01 a 386 & $(351 / 23,4)$ & 36,57 \\
\hline & 0,70 & & & 0,45 & \\
\hline 484,01 a 538 & $(511 / 14,4)$ & 23,70 & 386,01 a 464 & $(425 / 23,5)$ & 41,99 \\
\hline & 1,23 & & & 1,46 & \\
\hline 538,01 a 592 & $(551 / 12,6)$ & 11,01 & 464,01 a 542 & $(488 / 17,9)$ & 17,62 \\
\hline
\end{tabular}

Fuente: base PISA 2012. Promedio por quintil y desviación típica entre paréntesis; p= 0,000.

En el caso de Argentina los dos rangos de puntajes más bajos (quintiles 1 y 2), se encuentran alejados de la media; 3,36 y 1,87 desviaciones estándar respectivamente. En Chile, la distribución en torno a la media es relativamente simétrica y muestra diferencias moderadas. Los quintiles extremos ( 1 y 5 ) se ubican a algo más de una desviación estándar de la media, mientras los intermedios (2 y 4) se ubican a algo más de media desviación estándar. Es decir, que en lo que respecta al rendimiento en las pruebas Chile presenta diferencias más moderadas que Argentina.

Como complemento, el Cuadro 2 también muestra el porcentaje de estudiantes que concurren a escuelas pertenecientes a cada uno de los rangos de puntajes. En este sentido también Chile presenta una distribución algo más homogénea que Argentina. Mientras en el primer país algo más del 84 \% se ubica a menos de una desviación estándar de la media (quintiles 2 a 4) en el segundo lo hace algo más del $78 \%$. A su vez, en Chile la proporción de la población con desempeños por debajo de la media es algo menor que 
en Argentina. En el primer caso alcanza al 37,3 \% (rangos 1 y 2) y en el segundo al 40,38 $\%$ (rangos 1 a 3). Es decir, que en lo que hace al desempeño Chile muestra rendimientos algo superiores y más homogéneos que Argentina, mientras que este último país presenta rendimientos menores y con mayor desigualdad.

Finalmente, estimamos la relación entre el acceso al conocimiento escolarizado y la segregación según nivel social por escuela. Concretamente, en la tabla 3 calculamos el promedio de puntajes obtenidos en las pruebas en cada uno de los quintiles surgidos del porcentaje de padres con secundario terminado en cada escuela.

Tabla 3.

Relación entre Segregación Social y Desempeño en las Pruebas

\begin{tabular}{ccccc}
\hline \multirow{2}{*}{ Quintiles } & \multicolumn{2}{c}{ Chile } & \multicolumn{2}{c}{ Argentina } \\
\cline { 2 - 5 } & Promedio & $\begin{array}{c}\text { Desviaciones } \\
\text { a la media }\end{array}$ & Promedio & $\begin{array}{c}\text { Desviaciones a la } \\
\text { media }\end{array}$ \\
\hline 0-20 & 366 & & 339 & \\
& $(19,3)$ & $-1,07$ & $(40,5)$ & $-1,13$ \\
\hline 20,1-40 & 392 & & 360 & $-0,76$ \\
\hline & $(19,6)$ & $-0,57$ & $(50,9)$ & $-0,05$ \\
\hline 40,1 a 60 & 415 & & 400 & \\
\hline & $(30,6)$ & $-0,11$ & $(49,4)$ & 0,72 \\
\hline 60,1 a 80 & 434 & & 424 & 1,28 \\
\hline
\end{tabular}

Fuente: bases PISA 2012 - Desviación típica entre paréntesis; p= 0,000

La tabla 3 confirma la asociación entre el acceso al conocimiento y la segregación social. En ambos países los estudiantes en los quintiles más bajos obtuvieron puntajes de más de una desviación estándar por debajo de la media, mientras que los estudiantes en los más altos se ubicaron a más de una desviación por encima de ella. Así, en ambos países, la diferencia entre los extremos de la escala se ubica en torno a 2,5 desviaciones estándar. Sin embargo, debe recodarse que la distribución de la población presenta divergencias. Como lo indicaba la tabla 1, en Argentina se observa una mayor proporción o porcentaje de estudiantes con menor escolarización (quintiles 2 y 3 ) que alcanzan puntajes por debajo de la media en las Pruebas. En Chile se registra una mayor incidencia de grupos de mayor escolarización (quintiles 4 y 5) que alcanza puntajes más altos que el promedio.

En síntesis, las estimaciones que realizamos muestran patrones complejos. Desde el punto de vista de la segregación social, el sistema educativo chileno se caracteriza por la presencia de un grupo de estudiantes proporcionalmente pequeño pero altamente segregado que concurre a escuelas con progenitores con baja escolarización (mientras la mayoría asiste a escuelas con padres que accedieron a niveles educativos relativamente altos). Desde el punto de vista del acceso al conocimiento escolarizado, Chile parece haber logrado mayor acceso y mejores desempeños y más equitativos que la Argentina. 
Una mayor proporción de la población accede a la educación secundaria, y también una mayor y más equilibrada proporción de estudiantes obtiene puntajes altos en las Pruebas. Como contrapartida, Argentina presenta niveles o grados de segregación social más bajos que en Chile, pero el acceso a la educación es menor y el desempeño algo más heterogéneo. Es más pequeña la proporción de la población que finaliza la educación secundaria y también es más bajo y más desigual el puntaje que los estudiantes obtienen en las Pruebas. En definitiva, resulta un sistema más homogéneo en términos sociales, pero que ofrece menores garantías de permanencia en el sistema educativo y se presenta como algo más desigual en la distribución del saber escolarizado.

\section{Conclusiones}

En este estudio nos propusimos estimar la manera en que se configuran los patrones de segregación social en los sistemas educativos de Chile y Argentina, la relación que tienen estos con el acceso diferencial al conocimiento escolarizado y las proporciones de la población a la que estos afectan. La comparación entre ambos países reveló la existencia de patrones complejos. Concretamente, encontramos que si bien los grados (distancia a la media) de segregación social son menores en Argentina, afectan negativamente a una mayor proporción de la población. En Chile los grados de segregación social son mayores, pero afectan negativamente a menores proporciones de la población. A su vez, el sistema argentino ofrece menos garantías de permanencia (menores proporciones de la población concluyen la secundaria) y también menos garantías de un acceso igualitario al saber (el desempeño en las pruebas es menor y más heterogéneo). Chile ofrece más garantías de permanencia en el sistema (la graduación del nivel secundario es más alta) y la distribución del saber escolarizado es mayor y más homogénea. De manera que, al comparar los grados de segregación social tomando en cuenta las proporciones de la población afectada y el acceso al saber escolarizado, descubrimos patrones matizados. Puesto en apretada síntesis, en Chile parece existir un sistema educativo más heterogéneo o compartimentado socialmente (aislando, sobre todo, a grupos relativamente pequeños de la población con menor educación), pero que favorece un mayor y más equilibrado acceso al saber para el conjunto. En la Argentina, el sistema educativo se presenta como algo más homogéneo socialmente, pero con menos garantías de permanencia y de acceso equitativo al saber escolar

Además de comparar las distintas maneras en que se configura la segregación social y el acceso al conocimiento escolarizado en Chile y Argentina, un objetivo complementario de este estudio fue ver si esa configuración era compatible con la premisa que supone que Chile tiene niveles excepcionales de segregación social y de acceso diferencial al saber escolarizado, que resultarían de sus políticas de financiamiento educativo. Como ya aclaramos, los datos disponibles no nos permiten realizar una estimación estadística de la existencia de vínculos causales entre esas políticas y los niveles de segregación social o la desigualdad en el acceso al saber. Sin embargo, la comparación entre países con sistemas de financiación distintos puede indicar en qué medida la diferencia en los patrones de segregación y desigualdad en el acceso al saber es compatible o no con ese postulado.

En este sentido, la comparación relativiza la premisa de que Chile presenta niveles excepcionales de segregación social y acceso diferencial al conocimiento. En cambio, sugiere la importancia de ponderar las diversas dimensiones de la segregación social y el acceso al saber escolarizado a la hora de hacer comparaciones. Como señalamos, la 
segregación social en Chile es mayor que en Argentina cuando consideramos la diferencia de los sectores más segregados respecto al promedio, pero es menor cuando consideramos la proporción de la población negativamente afectada por ella. A su vez, en lo que hace al acceso al saber, la proporción de estudiantes por debajo de la media es mayor en Chile, pero la distancia a la misma de los sectores con menor puntaje es significativamente menor que en Argentina.

Así, la comparación que realizamos no permite probar que el sistema de financiamiento educativo basado en una lógica de mercado produce por sí mismo mayores grados de segregación pero que afectan negativamente a una menor proporción de la población que los sistemas que priorizan la educación pública y gratuita. Lo que sí indica es que el primer tipo de sistema no siempre o necesariamente segrega a mayores proporciones de la población que el primero. Asimismo, nuestras estimaciones no permiten probar que el sistema que privilegia la educación pública y gratuita y sin mecanismos de admisión, produce ciclos de escolarización más breves o mayor inequidad en la distribución del conocimiento. Sin embargo, sí podemos ver que esos sistemas no son incompatibles con este tipo de distribución; y que ésta puede ocurrir incluso en mayor medida que en sistemas basados en la lógica de mercado.

En suma, aunque la comparación que realizamos no conduce a conclusiones definitivas, sí indica que cuando se consideran las proporciones de la población afectada, y los vínculos entre la segregación social y la desigualdad en el acceso al saber, pueden revelarse patrones complejos. Y que tomar en cuenta esta complejidad permitiría, al menos potencialmente, una mayor comprensión de las desigualdades que se producen en los sistemas educativos y su potencial asociación con la política de financiación de la educación. En ese sentido, una última aclaración es que si bien, debido a los límites de extensión, hemos centrado el análisis en las formas de financiamiento educativo, es claro también que otros factores pueden concurrir en la constitución de un determinado patrón de segregación y desigualdad en el acceso al conocimiento. No ha habido espacio aquí para profundizar en ello, pero la diversidad de ciclos económicos y las tendencias opuestas en la movilidad social que mencionamos como parte de los factores contextuales en los que se constituyeron los patrones de segregación social y desigualdad en el acceso al conocimiento en Chile y Argentina, tienen una clara capacidad para influir en su configuración. Esta última observación habilita la hipótesis (aunque no la comprobación) de que, aún con sistemas de financiamiento que priorizan la lógica de mercado y los ciclos de expansión económica y reducción de la pobreza pueden asociarse a una mayor permanencia en el sistema educativo y mayor acceso al conocimiento escolarizado (aunque con un alto nivel de segregación a los grupos sociales con menor acceso a la educación). A su vez, ciclos económicos recesivos y con incremento de la pobreza como los que se dieron en la Argentina en las últimas décadas pueden asociarse a una menor permanencia en el sistema educativo y menor acceso al conocimiento escolarizado, aun cuando predomine la educación pública y gratuita y no se impongan los sistemas selectivos de admisión.

\section{Referencias}

Albornoz, F., Furman, M., Podestá, M. \& Razquin, P. (2015). Diferencias educativas entre escuelas privadas y públicas en Argentina, Desarrollo Económico, 56(218), 3-31.

Ascorra, P., López, V., Núñez, C., Bilbao, M., Gómez, G. \& Morales, M. (2016). Relación entre segregación y convivencia escolar en escuela públicas chilenas. Universitas Psychologica, 15(1), 65-78. 
Bellei, C. (2013). El estudio de la segregación socioeconómica y académica de la educación chilena. Estudios Pedagógicos, 39(1), 325-345. https://dx.doi.org/10.4067/ So718-07052013000100019.

Carabaña, J. (2015). La inutilidad de PISA para las escuelas, Julio Carabaña. Madrid: La Catarata.

Contreras, D., Sepúlveda, P. \& Bustos, S. (2010). When schools are the ones that choose: The effects of screening in Chile, Social Science Quarterly, 91(5), 1349-1368.

Duncan, O. \& Duncan, B. (1955). A methodological analysis of segregation indexes. American Sociological Review, 20, 210-217. https://doi.org/10.2307/2088328.

Dupriez, V. (2010). Methods of grouping learners at school. París: UNESCO.

Duro, E., Volpi, M, \& Contreras, D. (2010). La enseñanza secundaria en la región. Logros y desafíos. En E., Duro, (coord.), Educación secundaria derecho, inclusión y desarrollo. Desafíos para la educación de los adolescentes (pp. 156-178) Buenos Aires: UNICEF.

Falabella, A. (2015). El mercado escolar en Chile y el surgimiento de la nueva gestión pública: El tejido de la política entre la dictadura neoliberal y los gobiernos de centroizquierda (1979-2009). Educación Social, 36(132), 699-722.

Flores, C. \& Carrasco, A. (2013). (Des)igualdad de oportunidades para elegir escuela: Preferencias, libertad de elección y segregación escolar. Espacio Público 2 www. espaciopublico.cl.

Gasparini, L., Jaume, D., Monserrat, S. \& Vázquez, E. (2011). La segregación escolar en argentina. Documento de Trabajo 123 http://sedici.unlp.edu.ar/ handle/10915/3705.

Jaume, D. (2013). Un Estudio sobre el Incremento de la Segregación Escolar en Argentina. Documento de Trabajo Nro. 143. http://sedici.unlp.edu.ar/handle/10915/3705.

Krüger, N. (2014). Más allá del acceso: segregación social e inequidad en el sistema educativo argentino. Cuadernos de Economía, 33(63), 513-542.

Krüger, N. (2019). La segregación por nivel socioeconómico como dimensión de la exclusión educativa: 15 años de evolución en América Latina. Archivos Analíticos de Políticas Educativas, 27(8), 1-34, http://dx.doi.org/10.14507/epaa.27.3577.

Lieberson, S. (1981). An asymmetrical approach to segregation. En C. Peach (ed.), Ethnic segregation in cities. (pp. 61-83). Londres: Croom-Helm.

Martín, E. (2009). Currículo y evaluación estandarizada: colaboración o tensión. En E. Martin\& E. Martinez Rizo (comps.) Avances y desafíos en la evaluación educativa. (pp. 89-98) Buenos Aires: OEI/Fundación Santillana.

Martín, M. (2017). Inclusión y equidad: un análisis con base en el acceso y logros para el nivel medio de educación en Argentina. Semestre Económico, 20(43), 111-138.

Martínez García, J. (2016). La inutilidad de PISA para las escuelas. Revista Española de 
Sociología. 25(1), 153-156

Matelluna, H. (2018). La consolidación de prácticas de mercado en la educación chilena a partir de una política educativa des-mercantilizadora. Ponencia presentada al XVI Congreso Nacional Educación Comparada Tenerife. http://riull.ull.es/xmlui/ handle/915/12496.

Meyer, H., Benavot, A. (2013). PISA, power, and policy: the emergence of global educational governance. Oxford: Symposium Books.

Míguez, D., Dewey, M. (2017). The conditions of socioeconomic development. Exploring the legitimacy of social norms, trust, and corruption in Chile and Argentina. MPIfG Discussion Paper, 18(9), pp. 1-23, http://www.mpifg.de/pu/dp_abstracts/dp189.asp

Murillo, J. (2016). Midiendo la segregación escolar en América Latina. Un análisis metodológico utilizando el TERCE. Revista Iberoamericana sobre Calidad, Eficacia y Cambio en Educación, 14(4), 33-60. doi:10.15366/reice2016.14.4.002.

Moschetti, M. (2018). Alianzas público-privadas en educación. Un análisis de la política de subvenciones a escuelas privadas en barrios desfavorecidos de la Ciudad de Buenos Aires. Tesis de doctorado defendida en la Universidad Autónoma de Barcelona, Programa de Doctorado en Educación, Departamento de Pedagogía Sistemática y Social, Facultad de Ciencias de la Educación.

Narodowski, M. \& Nores, M. (2002). Socio-economic segregation with (without) competitive education policies. A comparative analysis of Argentina and Chile." Comparative Education, 38(4), 429-451.

Narodowski, M. \& Moschetti, M. (2015). The growth of private education in Argentina: evidence and explanations. Compare: a Journal of Comparative and International Education, 45(1), 47-69.

González Rozada, M., Narodowski, M., Gottau, V. \& Moschetti, M. (2015). De facto school choice and socioeconomic segregation in secondary schools of Argentina. https:// EconPapers.repec.org/RePEc:udt:wpecon:2015_05.

OCDE. (2016). Pisa 2015. Technical report. Paris: Organización para la Cooperación y el Desarrollo. http://www.oecd.org/pisa/data/2015-technical-report/.

Pereyra, M. Kotthoff, H.; Cowen,R. (2013). PISA a examen: cambiando el conocimiento, cambiando las pruebas y cambiando las escuelas. Introducción al monográfico. Profesorado. Revista de Currículum y Formación de Profesorado, 17(2), 6-14

Rigal, J.; Schoo, S.; Ambao, C. (2019). El ingreso a la escuela secundaria. Un análisis de los sistemas de distribución de vacantes en Argentina. Buenos Aires: Ministerio de Educación, Cultura, Ciencia y Tecnología.

Rivas, A. (2015). América Latina después de PISA. Buenos Aires: CIPPEC. https://www. cippec.org/publicacion/america-latina-despues-de-pisa-lecciones-aprendidas-dela-educacion-en-siete-paises-2000-2015/ 
Treviño, E., Valenzuela, J. P. \& Villalobos, C. (2016). Within-school segregation in the Chilean school system: What factors explain it? How efficient is this practice for fostering student achievement and equity? Learning and Individual Differences, $51,367-375$.

Valenzuela, J. P. (2008). Segregación en el sistema escolar chileno: en la búsqueda de una educación de calidad en un contexto de extrema desigualdad. En: Transformaciones del Espacio Público, II Escuela Chile-Francia. (pp. 131-156) Facultad de Arquitectura y Urbanismo, Universidad de Chile.

Valenzuela, J., Villalobos, C. \& Gómez, G. (2013). ¿Qué ha sucedido con los grupos medios? Los efectos de la elección escolar en la segregación socioeconómica en Chile: Un análisis georreferenciado. Espacio Público, Documento de referencia $N^{o}$ 3. https://www.espaciopublico.cl/segregacion-y-polarizacion-en-el-sistemaescolar-chileno-y-recientes-tendencias-que-ha-sucedido-con-los-grupos-medios

Valenzuela, J., Bellei, C. \& de los Ríos, D. (2009). Evolución de la segregación socioeconómica de los estudiantes chilenos y su relación con el financiamiento compartido. Proyecto FONIDE, No. 211 - 2006, Ministerio de educación de Chile, Universidad de Chile. https://scielo.conicyt.cl/scielo.php?script=sci_ arttext\&pid=So718-07052018000100157

Valenzuela, J. (2014). Socioeconomic school segregation in a market-oriented educational system. The case of Chile. Journal of Education Policy, 29(2), 217-241, doi: 10.1080/02680939.2013.806995

Veleda, C. (2014). Regulación y segregación educativa en la provincia de Buenos Aires. Archivos analíticos de política educativa.22 (42), 1-21.http://dx.doi.org/10.14507/ epaa.v22n42.2014

Verger, A., Bonal X. \& Zancajo, A. (2016). Recontextualización de políticas y (cuasi) mercados educativos. Un análisis de las dinámicas de demanda y oferta escolar en Chile. Archivos Analíticos de Políticas Educativas, 24(27), 2-27. http://dx.doi. org/10.14507/epaa.24.2098 